\title{
Properties and performance of SiPM based Cherenkov telescope for LHAASO
}

\author{
S. S. Zhang ${ }^{a *}$, M. J. Yang ${ }^{a}$, Z. Cao ${ }^{a b}$, Y. Zhang ${ }^{a}$, L. L. Ma ${ }^{a}$, L. Q. Yin ${ }^{a}$, Z. Y. You ${ }^{a b}$, M. \\ M. Ge ${ }^{c}$, L. Zhang ${ }^{c}$, R. Lu ${ }^{c}$, R. Zhou ${ }^{d}$, C. W. Yang ${ }^{d}$, T. Montaruli ${ }^{e}$, D. della Volpe ${ }^{e}, \mathbf{M}$. \\ Heller $^{e}$ for the LHAASO Collaboration \\ ${ }^{a}$ Institute of High Energy Physics, CAS, Beijing, China \\ ${ }^{b}$ University of Chinese Academy of Sciences, Beijing, China \\ ${ }^{c}$ Yunnan University, Kunming, Yunnan, China \\ ${ }^{d}$ Sichuan University, Chengdu, Sichuan, China \\ ${ }^{e}$ University of Geneva, Geneva, Switzerland \\ E-mail: zhangss@ihep.ac.cn
}

The Wide Field of View Cherenkov Telescope Array (WFCTA), one of the main component of LHAASO. It is composed by 18 imaging air Cherenkov telescopes, whose main scientific goal is to measure the ultra-high energy cosmic ray energy spectrum and composition from $30 \mathrm{TeV}$ to a couple of EeV. Each Cherenkov telescope is equipped with a SiPM-based camera composed by an array of $32 \times 32$ pixels, with angular size $0.5^{\circ} \times 0.5^{\circ}$, covering a field of view $16^{\circ} \times 16^{\circ}$. The SiPMs allow Cherenkov telescope to operate with the moon light thus increasing the duty cycle up to $30 \%$, much more than the $10 \%$ achieved by PMT-based telescope. The camera pixel is a square SiPM with photosensitive area of $15 \mathrm{~mm} \times 15 \mathrm{~mm}$ and with a cell size of $25 \mu \mathrm{m} \times 25 \mu \mathrm{m}$. The total active area of a WFCTA, instrumented with SiPMs, is about $4.15 \mathrm{~m}^{2}$, representing the first large-scale application of SiPM technology in cosmic ray experiments. The first WFCTA Cherenkov telescope was successfully put in operation at the January 2019 and a second one is being set-up at the LHAASO site in May 2019. More than 220,000 coincidence events of Cherenkov telescopes and the first pool of the water Cherenkov detector array (WCDA) have been collected. The properties and first performance of the telescope are described in this work.

36th International Cosmic Ray Conference -ICRC2019-

July 24th - August 1st, 2019

Madison, WI, U.S.A.

${ }^{*}$ Speaker. 


\section{Introduction}

Cosmic rays (CRs) have been discovered more than 100 years ago, but the origin of high energy CRs is still unknown. CRs energy spectrum, composition and anisotropy of CR directions are three basic observables for studying the origin, acceleration and propagation of CRs. Large High Altitude Air Shower Observatory (LHAASO) project [1,2] is designed to study the gamma ray astronomy from $40 \mathrm{GeV}$ to $1 \mathrm{PeV}$ and measure the $\mathrm{CR}$ energy spectrum and composition from 30 $\mathrm{TeV}$ to a couple of EeV. LHAASO consists of a $1 \mathrm{~km}^{2}$ EAS array (KM2A), a water Cerenkov detector array (WCDA), a wide field of view Cherenkov/fluorescence telescope array (WFCTA). KM2A includes 5195 scintillator detectors, with $15 \mathrm{~m}$ spacing, for electromagnetic particle detection and 1171 underground water Cherenkov tanks ( $36 \mathrm{~m}^{2}$ per tank), with $30 \mathrm{~m}$ spacing, for muon detection. WCDA has two $150 \mathrm{~m} \times 150 \mathrm{~m}$ water pools plus one $300 \mathrm{~m} \times 110 \mathrm{~m}$ pool. WCDA has total area of about 78,000 $\mathrm{m}^{2}$ and 3120 cells with a size of $5 \mathrm{~m} \times 5 \mathrm{~m}$, each equipped with an 8 -inches PMT. In the $150 \mathrm{~m} \times 150 \mathrm{~m}$ pool, a 1.5 inches PMT is placed near the 8-inches one to extend the dynamic range at higher energies, where the 8 -inches start to saturate. WFCTA, composed by 18 Cherenkov telescopes, main scientific goal is to measure the ultra-high energy cosmic ray energy spectrum and composition from $30 \mathrm{TeV}$ to a couple of EeV. Each Cherenkov telescope consists of an array of $32 \times 32$ Silicon photomultipliers (SiPMs) and a $5 \mathrm{~m}^{2}$ spherical aluminized mirror. It has a field of view (FOV) of $16^{\circ} \times 16^{\circ}$ with a pixel size of approximately $0.5^{\circ} \times 0.5^{\circ}$. The telescopes have been designed to be movable in order to allow switching between configurations to perform spectrum and composition measurements at different energies. SiPM-based Cherenkov telescopes can operate with moon-light, so the duty cycle of SiPM-based Cherenkov telescopes is three times as long as PMT-based telescopes. Two SiPM-based Cherenkov telescopes have been setup at the LHAASO site in May 2019.

\section{The Cherenkov telescope}

Each Cherenkov telescope (see Fig. 1(a)) consists of a SiPM camera (see Fig. 1(b)), a $\sim 5 \mathrm{~m}^{2}$ spherical aluminized mirror (see Fig. 1(b)), a removable and adjustable container, power supply system and slow control system. Mirrors are mounted at the back of the container. The $\mathrm{SiPM}$ camera is located at the focal plane which is $2870 \mathrm{~mm}$ away from the center of the mirror. Power supply system and slow control system are also installed inside the container with size of $4.2 \mathrm{~m} \times 2.5 \mathrm{~m} \times 2.6 \mathrm{~m}$. The container is mounted on a dump-truck frame with a pitching rotation system that allows the container to be lifted up-and-down manually, from $0^{\circ}$ to $90^{\circ}$ with a step of $0.1^{\circ}$. The mirror is made of 20 hexagon-shaped segments with side length of $297.1 \mathrm{~mm}$ and 5 half hexagon-shaped segments. The radius of each segment curvature is $5800 \mathrm{~mm}$ with a tolerance of $10 \mathrm{~mm}$. The reflectivity of the mirror is $\geqslant 82 \%$ for wavelength from $300 \mathrm{~nm}$ to $900 \mathrm{~nm}$.

The SiPM camera is a $32 \times 32 \mathrm{SiPMs}$ array which consists of 64 sub-clusters. Each sub-cluster (Fig. 2(a)) is composed by $4 \times 4$ SiPMs, each SiPM coupled with a light funnel (Winston cone), a pre-amplifier board, a voltage and temperature compensation loop board, two analogue boards, a digital board and a power regulator board. The SiPM used is a square Hamamatsu S14466 with photosensitive area of $15 \mathrm{~mm} \times 15 \mathrm{~mm}$. Each SiPM has 360,000 Geiger-APD cells with size $25 \mu \mathrm{m}$ [3]. The optical spot size is about $25.8 \mathrm{~mm} \times 25.8 \mathrm{~mm}$. A light funnel is use to funnel the 
photons of the optical spot on the SiPM. Each funnel has an entrance area of $25.8 \mathrm{~mm} \times 25.8 \mathrm{~mm}$ (including the funnel walls thickness) and an exit area of $15 \mathrm{~mm} \times 15 \mathrm{~mm}$ to match the SiPM area (Fig. 2(b)). SiPM signals are amplified by the pre-amplifier, and then are split into two channels, high gain and low gain, to get a good linearity over a wide dynamic range, to cover the corresponding 3.5 decades in the charge measurement. Finally, the signals are digitized by $50-\mathrm{MHz}$, 12 bits flash analog-to-digital-converters (FADCs) in the digital board and are collected by FPGAs to do further processing: single channel trigger, event trigger, signals transmission and storage etc. A temperature sensor is mounted on the back of each SiPM chip. The sensor is needed by the voltage and temperature compensation loop used to stabilize the SiPM gain against temperature variations. An optical filter window with cutoff wavelength of $550 \mathrm{~nm}$ is mounted in the front of the SiPM-based camera. The window improves the signal to noise ratio of the camera because the sky background light wavelength is dominated by wavelength $\geqslant 550 \mathrm{~nm}$ where SiPMs are still enough sensitive. The power consumption of each camera is about $760 \mathrm{~W}$. The camera is cooled by an internal self-circulating air-cooling system.

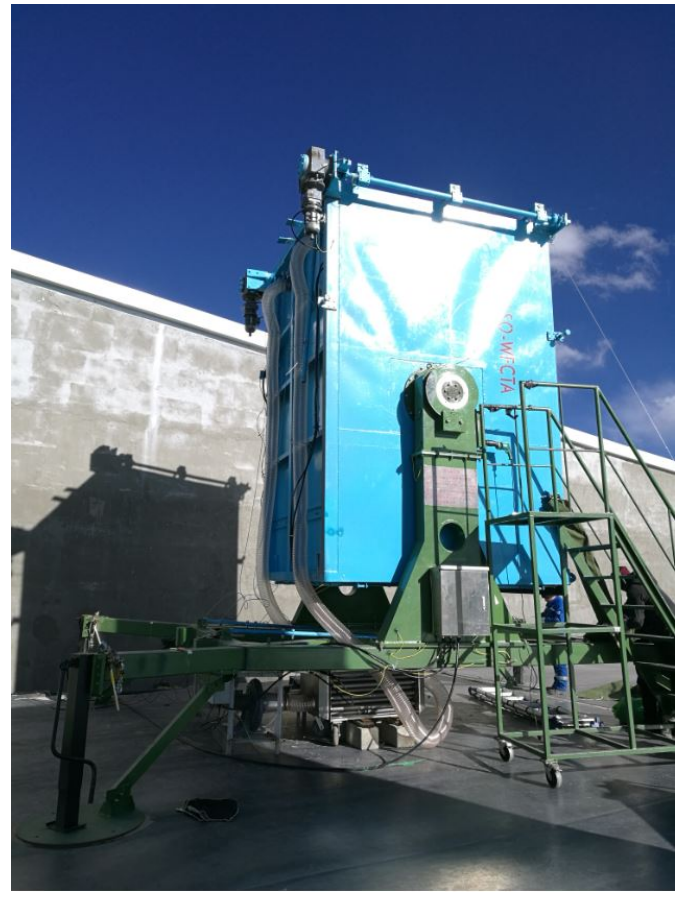

(a)

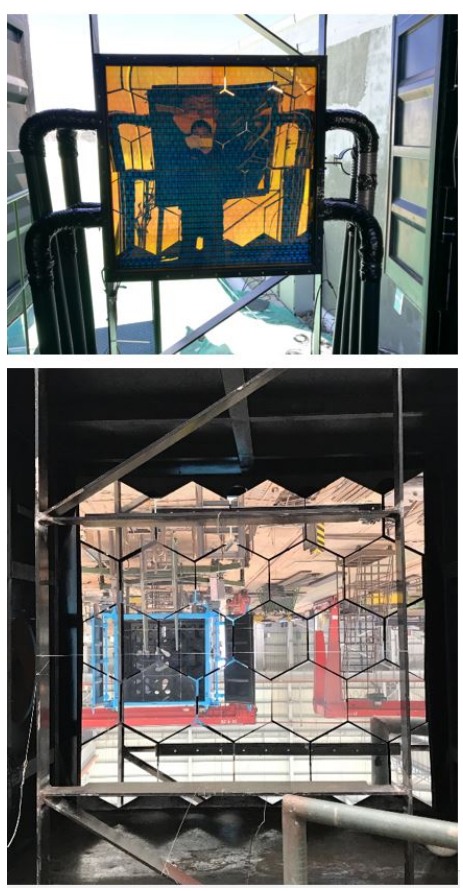

(b)

Figure 1: (a) A photo of the telescope. (b) Photos of the SiPM camera (up) and the mirror (down).

In the first observation stage, WFCTA aim to observe the primary energy range from $10 \mathrm{TeV}$ to $300 \mathrm{TeV}, 6$ Cherenkov telescopes will point at the zenith angle of $0^{\circ}$. In the second observation stage, WFCTA aim to observe the primary energy range from $30 \mathrm{TeV}$ to $10 \mathrm{PeV}, 6$ Cherenkov telescopes will point at the zenith angle of $30^{\circ}$ and each telescope point at different azimuth angles, in order to cover the sky with zenith angle from $22^{\circ}$ to $38^{\circ}$ and azimuth angle from $0^{\circ}$ to $180^{\circ}$. In the third observation stage, WFCTA aim to observe the primary energy range from $1 \mathrm{PeV}$ to 100 $\mathrm{PeV}$. To make sure that the position of shower maximum is far from the observation surface, the main axis of 12 Cherenkov telescopes will point at $45^{\circ}$ in zenith angle. Cherenkov telescopes will 


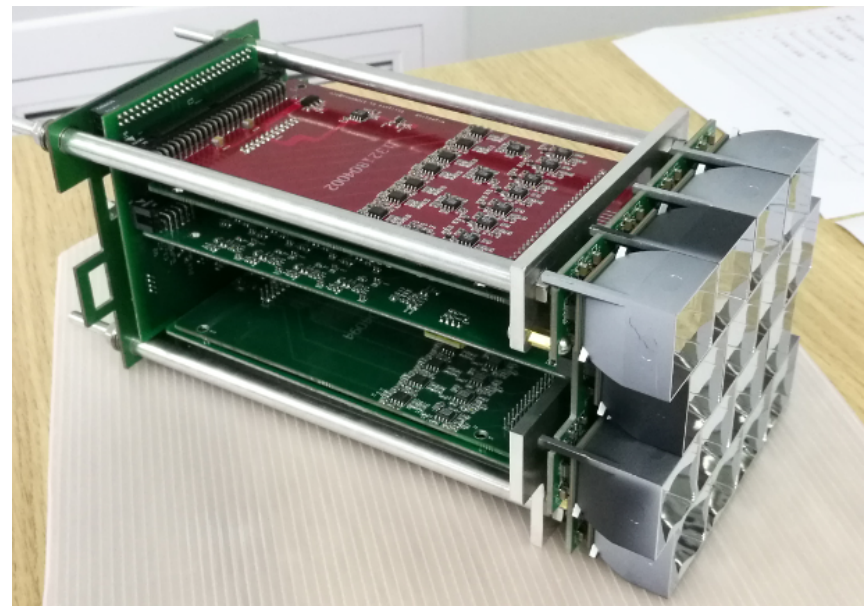

(a)

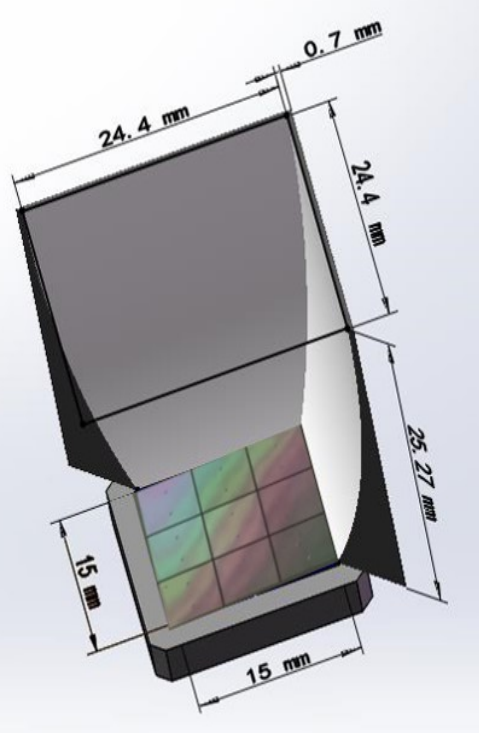

(b)

Figure 2: (a) A photo of one sub-cluster. (b) A half light funnel (Winston cone) and a SiPM.

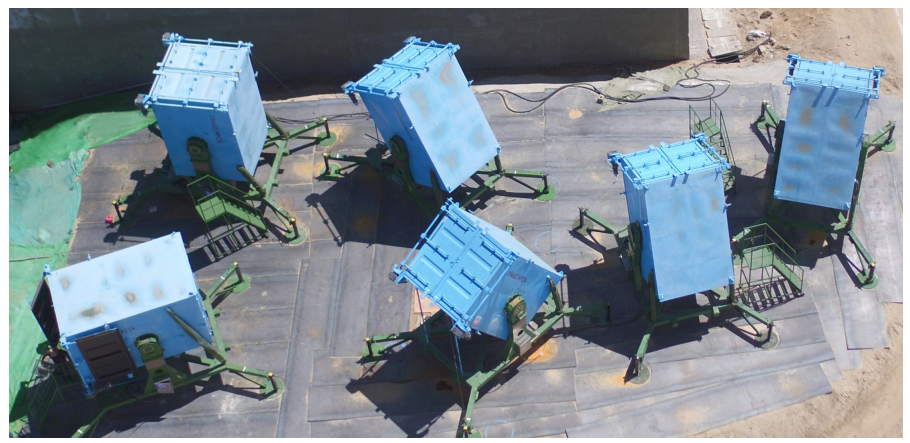

Figure 3: The layout of 6 Cherenkov telescopes.

point at different azimuth angles in order to cover $0^{\circ}$ to $360^{\circ}$ in azimuth. To extend the spectrum to higher energies $(\geq 100 \mathrm{PeV})$ and make a connection with experiments such as TA and Auger, the WFCTA is reconfigured to monitor the sky above the KM2A from a distance of $5 \mathrm{~km}$ for main detector array and $4 \mathrm{~km}$ for two slave detector arrays, by detecting shower fluorescence lights. The main detector array has 16 telescopes covering elevations from $3^{\circ}$ to $67^{\circ}$. The two slave detector arrays consists of 2 telescopes separately, covering elevations from $16^{\circ}$ to $32^{\circ}$.

\section{Performances and results of test run}

\subsection{Test run information}

Six removable and adjustable containers and mirrors have been installed in LHAASO site (Daocheng, $4410 \mathrm{~m}$ a.s.1., $600 \mathrm{~g} / \mathrm{cm}^{2}$ ) in March 2019 (see Fig. 2). At the end of January 2019, the first SiPM camera was installed and the first telescope began to work. In May 2019, a second 
SiPM camera was installed and the second telescope began to work. The two telescopes were in the southwest corner of the first pool of WCDA. The tow telescopes underwent night observations with and without moonlight, even run at a full moon night. All LHAASO detectors recorded the cosmic ray arrival time based on a White Rabbit Time System. More than 220,000 coincidence events of the telescopes and the WCDA first pool are collected. One of coincidence event is shown in Fig.4(a), Fig.4(b), Fig.4(c) and Fig.4(d).

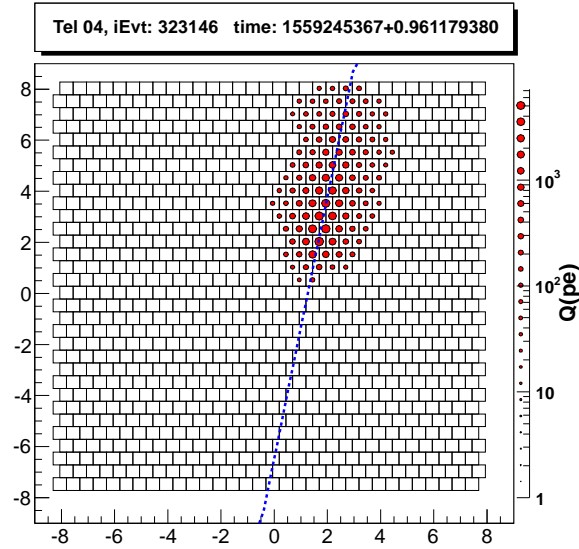

(a)

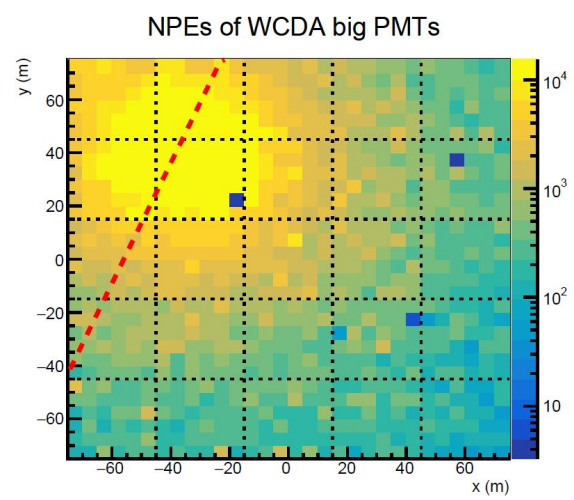

(c)

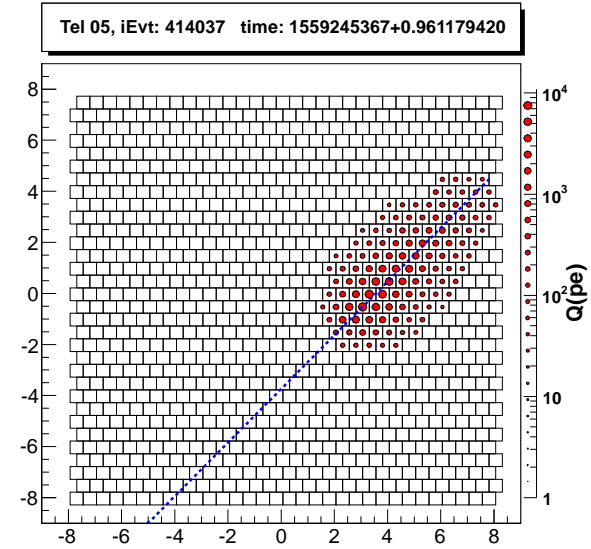

(b)

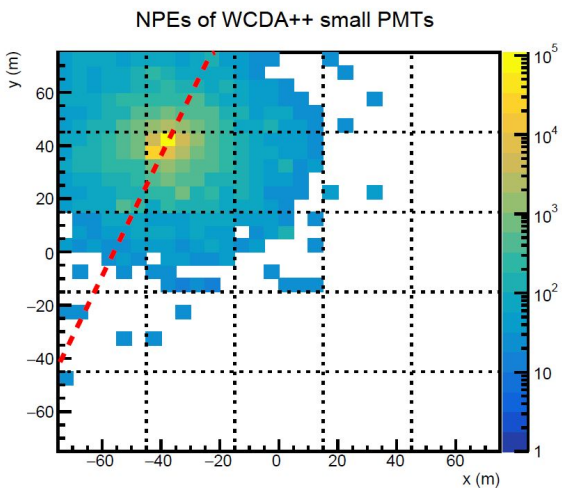

(d)

Figure 4: A coincidence event of two telescoopes and the WCDA first pool: (a) A Cherenkov event of first telescope, (b) A Cherenkov event of second telescope, (c) An event of the WCDA first pool using the 8-inchs PMT, (d) An event of the WCDA first pool using the 1.5-inchs PMT.

\subsection{Performances of SiPM Camera}

To monitor the gain of the SiPM camera, six UV-LEDs $(405 \mathrm{~nm})$ are mounted at the center of the mirror. The LED driving circuit and six LEDs work at the constant temperature of 30 ${ }^{\circ} \mathrm{C} \pm 0.2^{\circ} \mathrm{C}$. The instability of LED is less than $0.5 \%$ in different temperature [4]. A teflon diffuser is put in front of the LED to make the LED light uniform. Four LEDs are turned on every night. The LED will age after emitting light for a long time. The remaining two LEDs are used to monitor 
the four LEDs mentioned above, twice a month. All LEDs are calibrated by the absolute calibration device once a year.

Two $750 \mathrm{~W}$ blowers are used for the SiPM camera cooling. The temperature distribution of the SiPM camera is shown in Fig. 5(a). The difference between the highest and lowest temperatures is about $11^{\circ} \mathrm{C} \pm 0.2^{\circ} \mathrm{C}$. The temperature distribution on camera is within the adjustable range of high voltage and temperature compensation loops. The ambient temperature began to drop, and the temperature of SiPM also decreased during the night. The ambient temperature rises, and the temperature of SiPM rises as well during the day (Fig. 5(c) and Fig. 5(d)). The temperature difference at night is about $10^{\circ} \mathrm{C}$. High voltage and temperature compensation loops can keep the SiPM gain stable at different temperature environments. The gain of the SiPM camera is stabilized by high voltage and temperature compensation loops within $1 \%$ during the whole night (Fig. 5(b)).

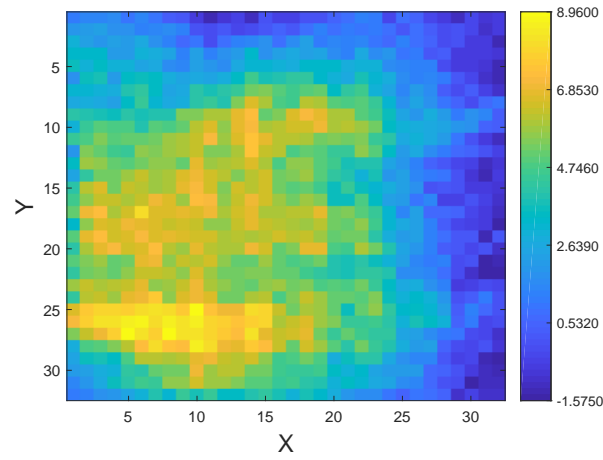

(a)

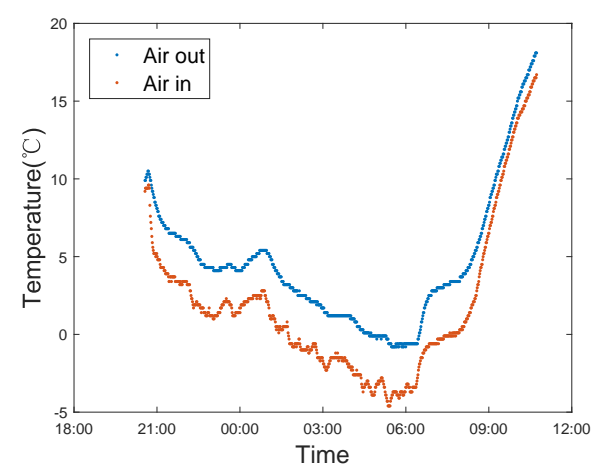

(c)

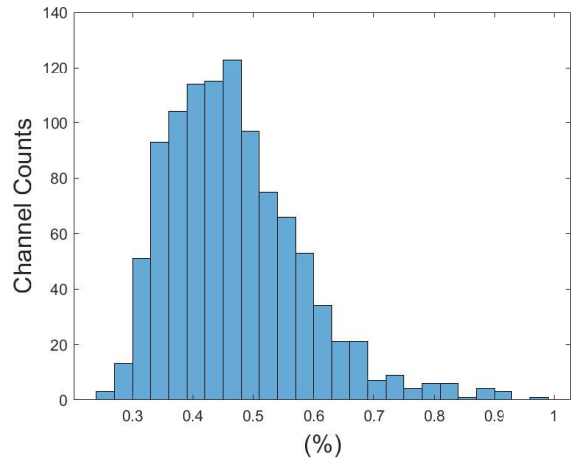

(b)

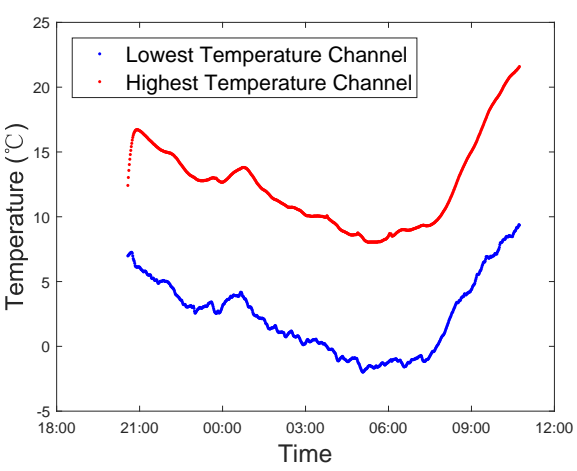

(d)

Figure 5: (a) Two-dimensional temperature distribution of $32 \times 32$ SiPMs array in the SiPM camera. (b) The $32 \times 32$ SiPMs gain instability distribution during the night is shown. The gain of the SiPM camera is stabilized by high voltage and temperature compensation loops within $1 \%$ during the whole night. (c) Two temperature sensors are put in the air in and the air out of the SiPM camera separately. The temperature of the two sensors as a function of time is shown. (d) The highest temperature position and lowest temperature position of the SiPM camera as a function of time is shown.

The gain of SiPM camera is stable in moonless night. A resistor of $\mathrm{R}=250 \Omega$ is put between the input hight voltage and SiPM cathode to protect the SiPM from damage under the strong light condition. The night sky background light induce a DC current in the SiPM, which induce a voltage 
drop on the bias resistor. Accordingly, the supply voltage, and then the gain, of the SiPM varies with the intensity of the sky background light. On April 19, 2019, the Cherenkov telescope were operated with the night of the full moon. The trajectory of the moon as a function of time is shown in Fig. 6(a). The space angle between one pixel (pixel 6) of the SiPM camera and the moon as a function of time is shown in Fig. 6(b). The baseline of SiPM is modulated by moonlight, the smaller the angle between SiPM and the moon, the higher the baseline (Fig. 6(c)). The gain of SiPM is linearly related to the intensity of moonlight on SiPM. The variation range of SiPM gain is within $\pm 2 \%$ during the full moon night (Fig. 6(d)). Baseline monitoring values can be used to correct the SiPM gain reduction due to moonlight offline. After correction, the SiPM gain is constant during the whole moon night (Fig. 6(e)).

\section{Discussion and Conclusion}

Two telescopes are successfully run at LHAASO site. More than 220,000 coincidence events with the WCDA first pool have been collected. The SiPM-based Cherenkov telescope has been shown to operate steadily on a full-moon night and achieve a longer duty cycle than PMT-based Cherenkov telescope. More detailed performance studies are under way, to validate the telescopes against the design requirements. A total of six Cherenkov telescopes will be put in operations by September 2019 and the complete array of 18 telescopes will be operational in LHAASO by the end of 2020.

\section{Acknowledgements}

This work is supported by the National Key R\&D Program of China No. 2018YFA0404201 and No. 2018YFA0404202, the Key Laboratory of Particle Astrophysics, Institute of High Energy Physics. The project No. 11475190 and No. 11675204 of NSFC also provide support to this study.

\section{References}

[1] Z. Cao et al., Chinese Physics C 34, 249 (2010)

[2] H.H. He et al., LHAASO Project: detector design and prototype, 31st ICRC, LODZ, (2009)

[3] Baiyang Bi et al., Nucl. Instr. and Meth. A 899, 94 (2018)

[4] M.J. Yang et al., The performance of LED calibration system for Cherenkov telescope of LHAASO, in Proceedings of 36th International Cosmic Ray Conference, Madison, WI, U.S.A. (2019) 


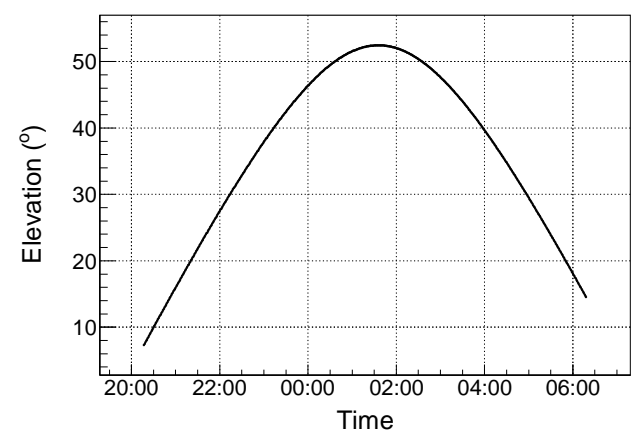

(a)

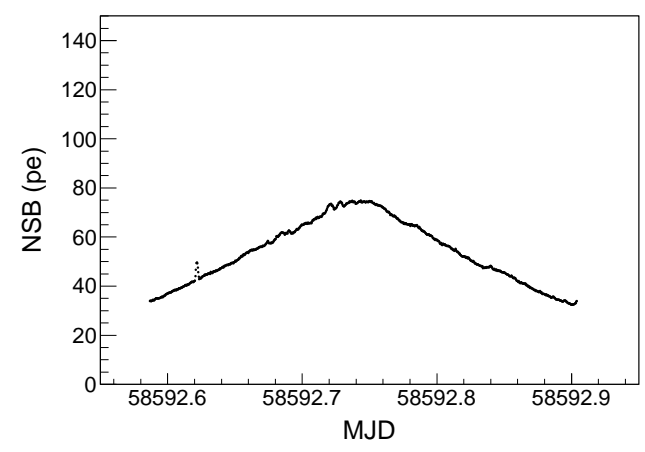

(c)

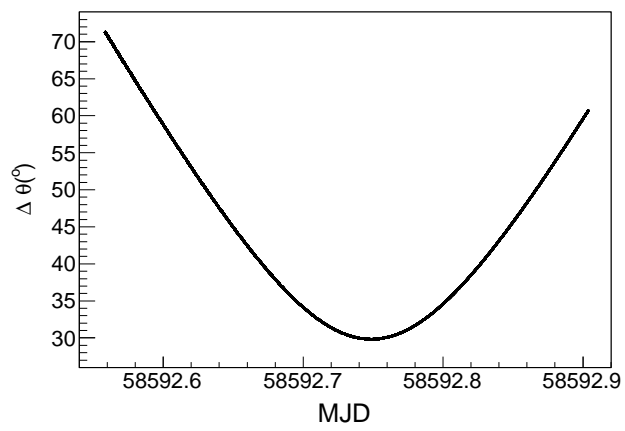

(b)

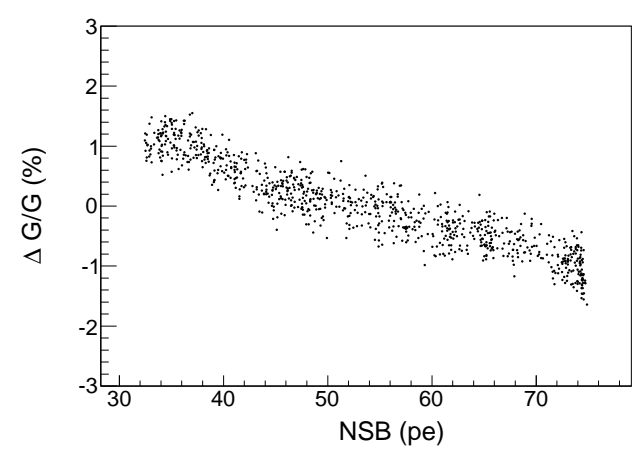

(d)

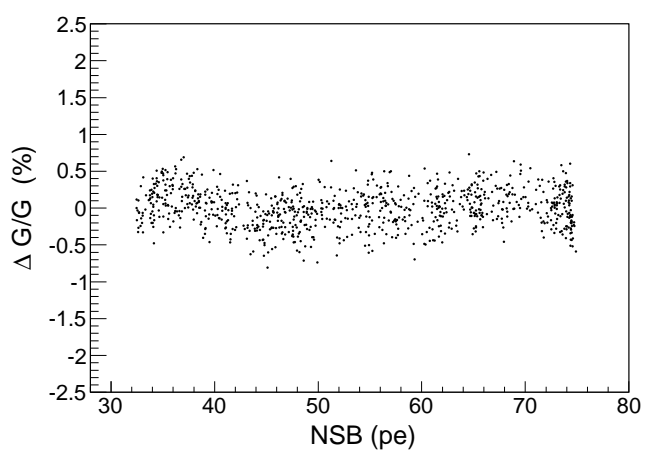

(e)

Figure 6: (a) The elevation of the moon as a function of time. (b) The space angle between one pixel (pixel 6) of the SiPM camera and the moon as a function of time. (c) The baseline measured by the one pixel of the SiPM camera as a function of time. (d) The gain of the one pixel as a function of the intensity of moonlight without the correction. (e) The gain of the one pixel as a function of the intensity of moonlight with the correction. 